\title{
Of Cheese and Crust: \\ A Proof of the Pizza Conjecture \\ and Other Tasty Results
}

\section{Rick Mabry and Paul Deiermann}

1. INTRODUCTION (SETTING THE TABLE). A pizza is divided into $2 N$ equiangular slices by means of $N$ straight, concurrent cuts, as in Figure 1 . It is then shared by two individuals ("Gray" and "White"), who alternate slices. When does the total area of the gray slices exceed that of the white slices?

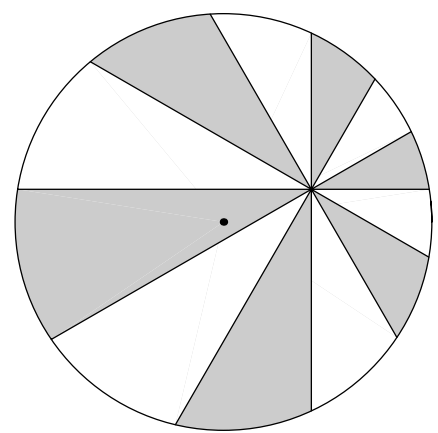

(a)

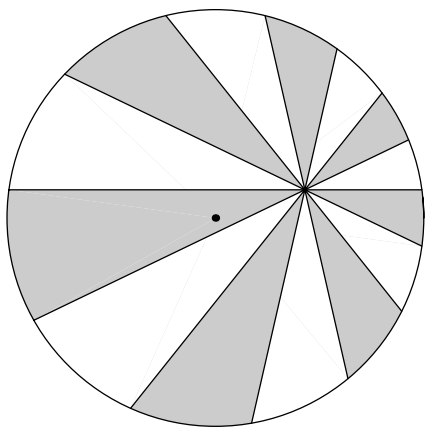

(b)

Figure 1. Two examples of equiangular sliced pizzas. (a) Gray area equals white when $N>2$ is even. (Here, $N=6$.) (b) Gray and white areas differ when $N$ is odd. (Here, $N=7$.)

The following theorem, which provides a precise solution to this question, had its genesis in a problem posed by L. J. Upton in Mathematics Magazine almost forty years ago.

Theorem 1 (Cheese Pizza Theorem). For a positive integer $N$, divide a pizza into $2 N$ slices by choosing an arbitrary point $P$ in the pizza and making $N$ straight cuts through $P$, the cuts meeting to form $2 N$ equal angles. Alternately color adjacent slices gray and white. Let $O$ denote the center of the pizza. The total areas of gray and white will then satisfy the following.

(a) When $N \geq 4$ is even, the gray area equals the white, but for all other $N$, gray equals white if and only if $O$ lies on a cut.

(b) If $O$ does not lie on a cut and $N=1, N=2$, or $N$ is odd with $N \equiv 3(\bmod 4)$, then gray exceeds white if and only if $O$ lies in a gray slice.

(c) If $O$ does not lie on a cut and $N$ is odd with $N \geq 5$ and $N \equiv 1$ ( $\bmod 4)$, then white exceeds gray if and only if $O$ lies in a gray slice.

Thus if the disk is a uniformly distributed cheese pizza shared by gray and white, then for even $N \geq 4$, gray and white get equal amounts. When $O$ lies on a cut, gray and white share equally by symmetry. Therefore we will henceforth assume that $O$ 
does not lie on a cut, but lies within a gray slice, as in Figure 1. In that case, when $N=2$ or is odd, gray will never equal white, and we will know which set of slices to choose to get the greater amount of cheese: if $N=2,3,7,11, \ldots$, then gray will get more; if $N=5,9,13, \ldots$, then white will win.

When $N=1$ the situation is completely clear. When $N=2$, the result seems pretty obvious, but Figure 2 provides a "proof with few words." The results for even $N$ have been known for some time. Our contribution to the Cheese Pizza Theorem will be to settle the case for odd $N \geq 3$-the "Pizza Conjecture" (as it is known in some circles). For dessert, baked into our proof is a theorem of sufficient generality to let us slice up some more exotic dishes.
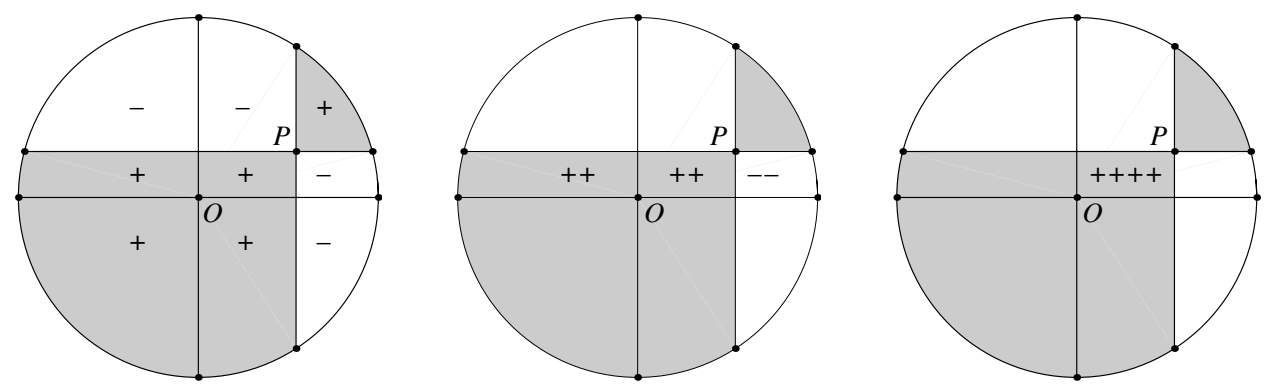

Figure 2. For $N=2$, gray minus white $=4 \times$ rectangle. Proof. Move signs from bottom to top, then from left to right.

The results for even $N \geq 4$ are sprinkled throughout the literature, and seem to make their first appearance with a solution by Goldberg [6] to a problem proposed by Upton in [16] (see also [7], [8], [13], [14], [1], [2], and [9]). Proofs for even $N \geq 4$ are accomplished by calculating exact areas of certain sets of pairs of opposite slices. In [4] the case for $N=4$ is illustrated by Carter and Wagon as a "proof without words" using a dissection of the white and gray slices into sets of pairwise congruent regions.

For odd $N$, the total areas of the gray slices and the white slices are shown to be unequal by an elegant continuity argument of Don Coppersmith (see [4]), utilizing the transcendence of $\pi$. However, Coppersmith's proof does not tell us which total area is greater. In [3] Carter, Duncan, and Wagon proposed the problem for the cases $N=3$ and 5 . Though it is not stated there, they made the general conjecture for all odd $N \geq 3$, observing that the location of the center alone determines which set of slices has greater area. Stanley Rabinowitz mentions this in exercise 26.3 of his asyet-unpublished manuscript [15], and it is also stated in [11] (a book of intriguing mathematical mysteries), in the section titled "The Pizza Problem."

In [5] solutions for the cases $N=3$ and 5 are given. The main idea is the comparison of areas of certain sets of "strips" associated with the slices. This trick is "kneaded" in the present paper, eventually rising to help complete the theorem for odd $N$.

Our proof of the Pizza Conjecture will amount to calculating gray minus white and will consist of several parts. First we show that differences of areas of opposite slices can be expressed in terms of areas of two "central strips" (see Figures 4 and 5) and such strips have an easy area formula we call a "strip function" $s(r)$, where $r$ is the thickness of the strip. Using these strips we then find a fairly simple expression for the difference between the total areas of gray and white slices, reducing the pizza problem to an inequality having an alternating sum involving $s(r)$. Fortunately, $s(r)$ has such a convenient power series expansion that we are able to further reduce the problem to solving the inequality when the function $s(r)$ is replaced by the function 
$r^{n}$, where $n \geq 3$ is odd. Unfortunately, the argument of $s$ takes the form of a sum of sine functions, so we have to deal with binomial expansions of these sums, leading to complicated sums of powers of sines. The good news is that applying standard manipulations (involving complex exponential functions and a geometric sum of roots of unity) to these powers of sines yields a sum that contains a product of two alternating lacunary binomial sums. But the great news is that these alternating sums turn out to represent the number of certain lattice paths in the plane and hence are never negative. So at the end we travel along lattice paths to deliver the pizza. And it gets even better, because the methods here that solve the pizza problem also make it possible to handle some specialty menu items-similar divisions of hemispheres, cones, and other figures. We discuss these in Section 4.

2. SLICES AND STRIPS (THE WAYS OF PARTING). When we make an odd number of cuts through $P$ on our pizza, we see that opposing slices lie in differently colored sets. That is, if points $X, P, Y$ are collinear with $X$ and $Y$ in different slices, then those slices have different colors. In this section we will obtain a little result (the "Opposing Slices Theorem") that will aid us in computing the difference of the areas of opposing slices, as opposed to computing certain sums of areas of such slices, the main tool in the case for even $N$.

First we sharpen the cutlery by agreeing on a few definitions and conventions. The length of an $\operatorname{arc} \mathcal{C}$ will be denoted $|\mathcal{C}|$, and similarly, the area of a region $\mathcal{R}$ will be noted by $|\mathcal{R}|$. If we claim that two regions meet, then we mean that their intersection contains an open set. We will not always explicitly distinguish regions from their areas if it is clear from the context.

Let our pizza be the unit disk, $O$ denoting the origin. Then all of our radii, sectors, arcs, etc., will implicitly involve a unit radius. Let $\hat{\mathbf{r}}(\theta)$ denote the unit radial vector in the direction of $\theta$, that is, $\hat{\mathbf{r}}(\theta)=\langle\cos \theta, \sin \theta\rangle$. For a point $P$ in the plane, let $\operatorname{ray}(P, \theta)$ denote the ray emanating from a point $P$ in the direction of $\hat{\mathbf{r}}(\theta)$, that is, $\operatorname{ray}(P, \theta)=$ $\{P+t \hat{\mathbf{r}}(\theta) \mid t \geq 0\}$.

For $P$ in the unit disk and $\beta<\gamma$, define the slice at $P$ from $\beta$ to $\gamma$ to be the interior of the region in the unit disk swept out by $\operatorname{ray}(P, \theta)$ as $\theta$ increases from $\beta$ to $\gamma$. The opposing slice of this slice shall mean the slice at $P$ from $\pi+\beta$ to $\pi+\gamma$. A sector is simply a slice with $P=O$. (Some authors refer to slices as pseudo-sectors, but this is pizza.)

All lines and line segments should be considered to be directed. For a line (respectively, chord) $L$ that does not contain the origin, let $L^{\prime}$ denote the line (respectively, chord) parallel to $L$ and passing through the origin. When such an $L$ intersects the unit circle we define the (central) strip generated by $L$, denoted by $\operatorname{str}(L)$, to be the region in the disk lying between $L$ and $L^{\prime}$.

We also employ the following notation. For $P$ in the disk, if $B$ and $C$ are the respective points where $\operatorname{ray}(P, \beta)$ and $\operatorname{ray}(P, \gamma)$ intersect the circle, we let $P \overparen{B C}$ denote the slice at $P$ from $\beta$ to $\gamma$. (See Figure 3.) Similarly, for a line $L$ that intersects the circle but does not contain the origin, if $\overline{B C}$ and $\overline{B^{\prime} C^{\prime}}$, both parallel to $L$ with $\overline{B^{\prime} C^{\prime}}$ containing the origin, are the respective directed chords formed by intersecting $L$ and $L^{\prime}$ with the circle, then we denote $\operatorname{str}(L)$ by $\overparen{B^{\prime} B} \widehat{C C^{\prime}}$. (See Figure 4.)

Now we state and prove the aforementioned theorem, which essentially says that we can evaluate the difference of areas of opposing slices by adding or subtracting areas of two related strips.

Theorem 2 (Opposing Slices Theorem). Let two chords $L$ and $M$ intersect at a single point $P$ within the disk and let $S$ and $T$ be a pair of opposing slices formed by 


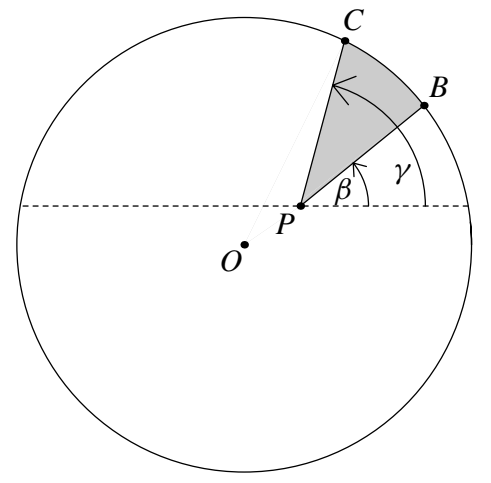

Figure 3. A slice: slice at $P$ from $\beta$ to $\gamma=P \overparen{B C}$.

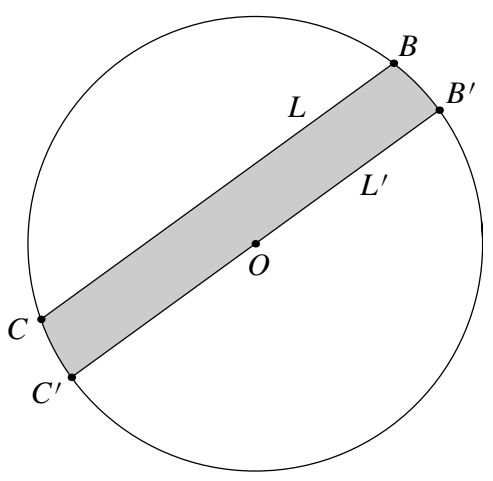

Figure 4. A strip: $\operatorname{str}(L)=\overparen{B^{\prime} B C C^{\prime}}$.

these chords. Then the difference $|T|-|S|$ in their areas is the sum of the areas of those of the two strips $\operatorname{str}(L)$ and $\operatorname{str}(M)$ that meet $T$ minus those that meet $S$.

Proof. The result is clear from the pictures in Figure 5. The two images show two cases that are fundamentally different, namely, that

(a) the origin is contained in one of the two slices, or

(b) the origin is contained in neither slice.

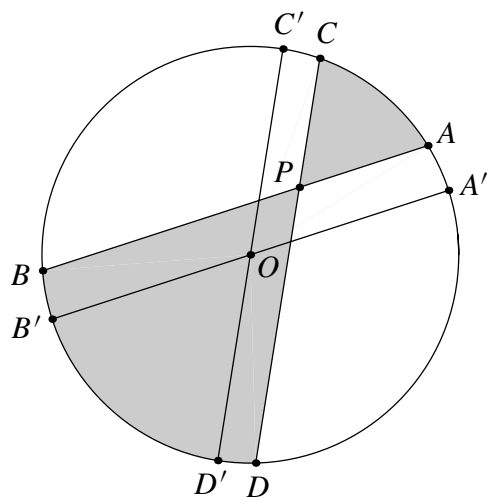

(a)

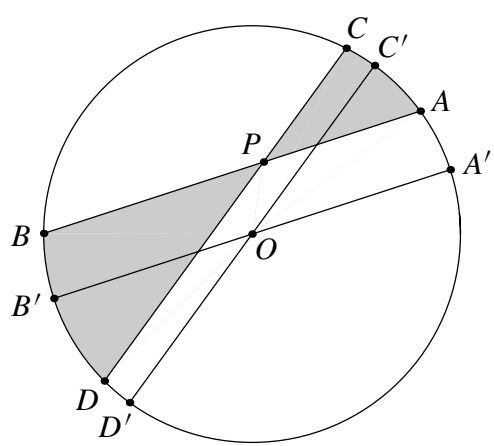

(b)

Figure 5. Opposing slices. (a) Origin in a slice. (b) Origin in neither slice.

Let $L=\overline{A B}$ and $M=\overline{C D}$ be such that $S=P \overparen{A C}$ and $T=P \overparen{B D}$. Let $L^{\prime}=\overline{A^{\prime} B^{\prime}}$ and $M^{\prime}=\overline{C^{\prime} D^{\prime}}$ be directed diameters parallel to $\overline{A B}$ and $\overline{C D}$, respectively.

In case (a), both strips $\operatorname{str}(L)$ and $\operatorname{str}(M)$ meet the slice containing the origin, while neither meets the opposite slice. And indeed we have

$$
\begin{aligned}
|T|-|S| & =|P \overparen{B D}|-|P \overparen{A C}| \\
& =\left|\widehat{A^{\prime} A} \overparen{B B^{\prime}}\right|+\left|\widehat{C^{\prime} C} \widehat{D D^{\prime}}\right|+\left|O \widehat{B^{\prime} D^{\prime}}\right|-\left|O \widehat{A^{\prime} C^{\prime}}\right| \\
& =|\operatorname{str}(L)|+|\operatorname{str}(M)|,
\end{aligned}
$$


where the final equality holds since the two opposite sectors $O \widehat{B^{\prime} D^{\prime}}$ and $O \overparen{A^{\prime} C^{\prime}}$ have the same area. Thus, in case (a) we have that the difference of the slices is the sum of the strips.

In case (b), each strip meets a single slice and we have, similarly,

$$
|T|-|S|=|\operatorname{str}(L)|-|\operatorname{str}(M)|,
$$

so, in case (b) we have that the difference of the slices is the corresponding difference of the strips.

Figure 5 does not include all possible configurations of opposing slices. The reader can easily verify the details concerning the other configurations, which are illustrated in Figure 6.
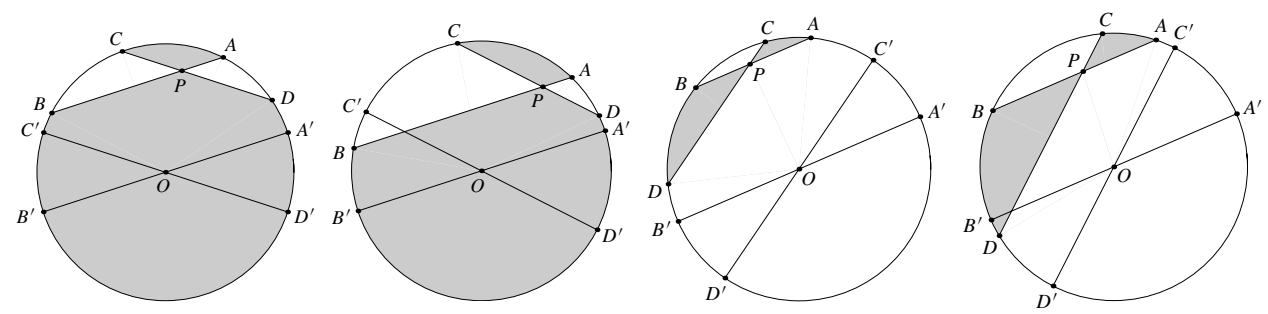

Figure 6. Opposing slices, remaining subcases.

3. PROOF OF THE PIZZA CONJECTURE. We now prove Theorem 1 for odd $N$. Using the Opposing Slices Theorem we write a simple formula for $\Delta S$, which will denote the gray minus the white areas of the two sets of slices. For a fixed point $P$ in the disk and for each $j=0,1,2, \ldots, 2 N$, let $Q_{j}$ denote the point of intersection of the circle and $\operatorname{ray}(P, j \pi / N)$, where $Q_{0}=Q_{2 N}$. The "cuts" of the pizza are the $N$ line segments $C_{j}=\overline{Q_{j} Q_{N+j}}, j=0,1, \ldots, N-1$, which are concurrent at $P$, cyclically consecutive cuts meeting at equal angles of $\pi / N$ radians. With no loss of generality, we take $P=R \hat{\mathbf{r}}(\alpha)$, where $0<R \leq 1$ and $0<\alpha<\pi / N$. This puts the origin in the interior of $P \widehat{Q_{N} Q_{N+1}}$.

Let $A_{j}=\left|P \widehat{Q_{j} Q_{j+1}}\right|, j=0,1, \ldots, 2 N-1$. Then

$$
\Delta S=\sum_{j=0}^{2 N-1}(-1)^{j-1} A_{j}=\sum_{j=0}^{N-1}(-1)^{j}\left(A_{N+j}-A_{j}\right) .
$$

This is the alternating sum of the areas of cyclically consecutive slices taken counterclockwise about $P$, also expressed as an alternating sum of differences of opposing slices. The signs are chosen so the area $A_{N}$ of the slice containing the origin is added.

We note that our restriction $R>0$ precludes $P$ from being the origin, and $0<$ $\alpha<\pi / N$ negates the possibility of $O$ lying on one of the cuts $C_{0}$ or $C_{1}$. But we may dispense with these possibilities, since we are assuming that $O$ does not lie on a cut.

So now our aim becomes clear: we must prove that $\Delta S>0$ for $N=3,7,11, \ldots$ and $\Delta S<0$ for $N=5,9,13, \ldots$, or more succinctly,

$$
(-1)^{(N-1) / 2} \Delta S<0, \quad \text { for all odd } N \geq 3 \text {. }
$$




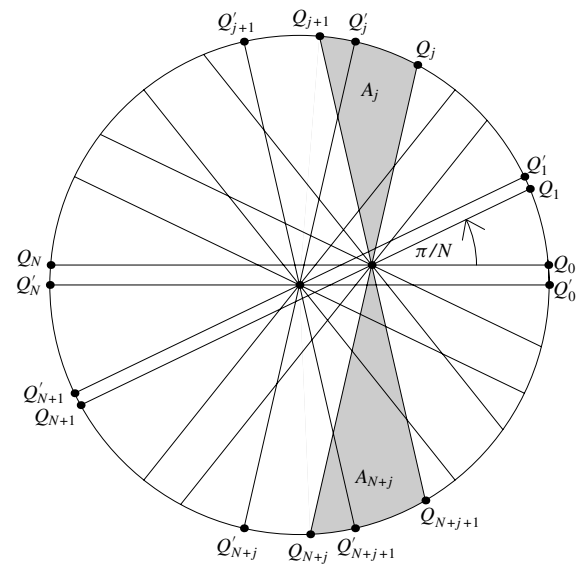

Figure 7. Opposing slices $P \widehat{Q_{j} Q_{j+1}}$ and $P \widehat{Q_{N+j} Q_{N+j+1}}$.

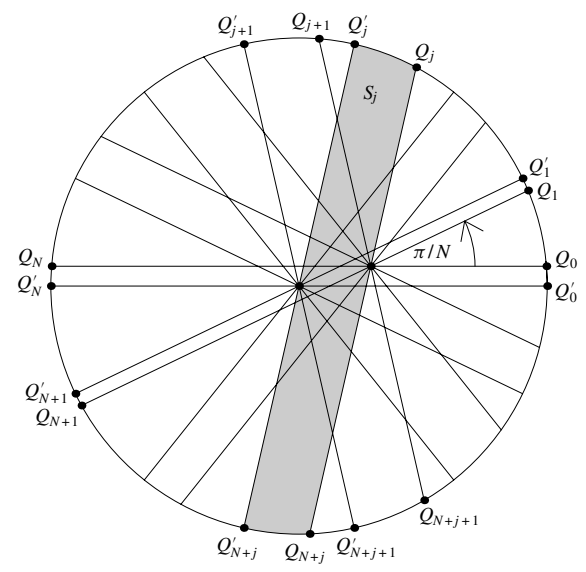

Figure 8. Strip $\widehat{Q_{j} Q_{j}^{\prime}} \widehat{Q_{N+j}^{\prime} Q_{N+j}}$.

The Opposing Slices Theorem involves central strips, which invoke central cuts $C_{j}^{\prime}$ through $O$ and parallel to the cuts $C_{j}$. We then let $S_{j}=\left|\operatorname{str}\left(C_{j}\right)\right|$, for $0 \leq j \leq N$, with $S_{0}=S_{N}$. In view of the theorem, we have that $A_{N}-A_{0}=S_{0}+S_{1}$, while for $1 \leq j<$ $N$ we have $A_{N+j}-A_{j}=-\left(S_{j}-S_{j+1}\right)$. This now implies, along with equation (1), that

$$
\Delta S=S_{0}+S_{1}+\sum_{j=1}^{N-1}(-1)^{j-1}\left(S_{j}-S_{j+1}\right)=2 \sum_{j=1}^{N}(-1)^{j-1} S_{j}
$$

Notice $\Delta S$ ends up being an alternating sum of areas of cyclically consecutive strips, having started out as an alternating sum of areas of slices in (1).

Let $r \leq 1$ denote the distance from a chord $L$ to the origin (i.e., the width of $\operatorname{str}(L))$, and let $s(r)=|\operatorname{str}(L)|$; we will call $s$ the strip function for cheese pizza. For $1 \leq j \leq N$, we let $r_{j}$ be the distance from the origin to the cut $C_{j}$, which is readily calculated as $r_{j}=R \sin (j \pi / N-\alpha)$. It follows that $S_{j}=s\left(r_{j}\right)$, and if we let $d=R \csc (\pi / N) \sin (\alpha)$ and $w=R \csc (\pi / N) \sin (\pi / N-\alpha)$, a routine use of trigonometric identities leads to

$$
\begin{aligned}
\Delta S & =2 \sum_{j=1}^{N}(-1)^{j-1} s\left(R \sin \left(\frac{j \pi}{N}-\alpha\right)\right) \\
& =2 \sum_{j=1}^{N}(-1)^{j-1} s\left(d \sin \left(\frac{(j-1) \pi}{N}\right)+w \sin \left(\frac{j \pi}{N}\right)\right) .
\end{aligned}
$$

A straightforward calculation shows that

$$
s(r)=\int_{0}^{r} 2 \sqrt{1-y^{2}} d y .
$$

In terms of Maclaurin series, we have $\sqrt{1-y^{2}}=1-\frac{1}{2} y^{2}-\frac{1}{8} y^{4}-\cdots$, which for us 
has the important implication that

$$
s(r)=2 r+\sum_{\substack{n=3 \\ n \text { odd }}}^{\infty} c_{n} r^{n}, \quad \text { with each } c_{n}<0 .
$$

Symmetry of the sine function shows that

$$
\sum_{j=1}^{N}(-1)^{j-1} \sin \left(\frac{j \pi}{N}\right)=\sum_{j=1}^{N}(-1)^{j-1} \sin \left(\frac{(j-1) \pi}{N}\right)=0 .
$$

Thus when we apply the expansion (4) of $s(r)$ to the summand in $\Delta S$ in (2), the total contribution due to the linear term is zero. We get, after switching the order of summations and employing the binomial theorem, that

$$
\begin{aligned}
(-1)^{\frac{N-1}{2}} \Delta S & =(-1)^{\frac{N-1}{2}} \sum_{\substack{n=3 \\
n \text { odd }}}^{\infty} 2 c_{n} \sum_{j=1}^{N}(-1)^{j-1}\left[w \sin \left(\frac{j \pi}{N}\right)+d \sin \left(\frac{(j-1) \pi}{N}\right)\right]^{n} \\
& =(-1)^{\frac{N-1}{2}} \sum_{\substack{n=3 \\
n \text { odd }}}^{\infty} 2 c_{n} \sum_{k=0}^{n}\left(\begin{array}{l}
n \\
k
\end{array}\right) w^{k} d^{n-k} \sum_{j=1}^{N}(-1)^{j-1} \sin ^{k}\left(\frac{j \pi}{N}\right) \sin ^{n-k}\left(\frac{(j-1) \pi}{N}\right) \\
& =\sum_{\substack{n=3 \\
n \text { odd }}}^{\infty} 2 c_{n} \sum_{k=0}^{n}\left(\begin{array}{l}
n \\
k
\end{array}\right) w^{k} d^{n-k} W(N, n, k),
\end{aligned}
$$

where we have let

$$
W(N, n, k)=(-1)^{\frac{N-1}{2}} \sum_{j=1}^{N}(-1)^{j-1} \sin ^{k}\left(\frac{j \pi}{N}\right) \sin ^{n-k}\left(\frac{(j-1) \pi}{N}\right) .
$$

When $k=0$ or $k=n$ we have $W(N, n, k)=0$, again by symmetry. For the remaining cases, it turns out that the sign of $W(N, n, k)$ is, amazingly, independent of $N, n$, and $k$. We can now solve the Pizza Conjecture by proving the following theorem.

Theorem 3 (Pizza Sign Theorem). For $N \geq 3, n \geq 3$, both odd, and $0<k<n$,

$$
W(N, n, k) \geq 0
$$

with $W(N, n, k)=0$ if and only if $n<N$.

We let $W$ denote $W(N, n, k)$ in what follows, as we break up the proof into two lemmas. Let us adopt a standard convention of binomial coefficients, namely that $\left(\begin{array}{l}a \\ b\end{array}\right)=0$ if $b<0$ or $a<b$. The first lemma rewrites $W$ as a beautiful sum of products (of sums).

Lemma 1 (Pizza Bi-Product Lemma). If $n<N$, then $W=0$, and if $n \geq N$, then

$$
W=\frac{N}{2^{n-1}} \sum_{\substack{q \in(0, N) \\
q \neq k(\bmod 2)}} \sin \left(\frac{\pi q}{N}\right) \sum_{u \in \mathbb{Z}}(-1)^{u}\left(\begin{array}{c}
k \\
\frac{k-q+N}{2}-u N
\end{array}\right) \sum_{v \in \mathbb{Z}}(-1)^{v}\left(\begin{array}{c}
n-k \\
\frac{n-k-q}{2}-v N
\end{array}\right) .
$$


The proof of Lemma 1 is outlined in the Appendix. The case $W=0(n<N)$ of Theorem 3 is proven there, so we will henceforth assume that $n \geq N$. Since $\sin \left(\frac{\pi q}{N}\right)>$ 0 for all $q \in(0, N)$, to finish Theorem 3 , we need only apply the following result twice.

Lemma 2 (Lattice Lemma). Let $A, B$, and $N$ be positive integers. Let $\mathcal{L}(A, B)$ denote the number of lattice paths from $(0,0)$ to $(A, B)$ which do not intersect the lines $y=x \pm N$, with $|A-B| \leq N$. Then

$$
\mathcal{L}(A, B)=\sum_{u \in \mathbb{Z}}(-1)^{u}\left(\begin{array}{c}
A+B \\
A-u N
\end{array}\right) .
$$

In particular, the sum in (7) is never negative. Here, for $A, B>0$, a lattice path from $(0,0)$ to $(A, B)$ is defined to be a path consisting of integral steps in either the positive $x$ or positive $y$ directions. See Figure 9 for illustrations of lattice paths. It is clear that the admissible paths in the lemma, namely the ones that do not touch the lines $y=x \pm N$, are precisely the ones that satisfy $|x-y|<N$ for all $(x, y)$ on the path.
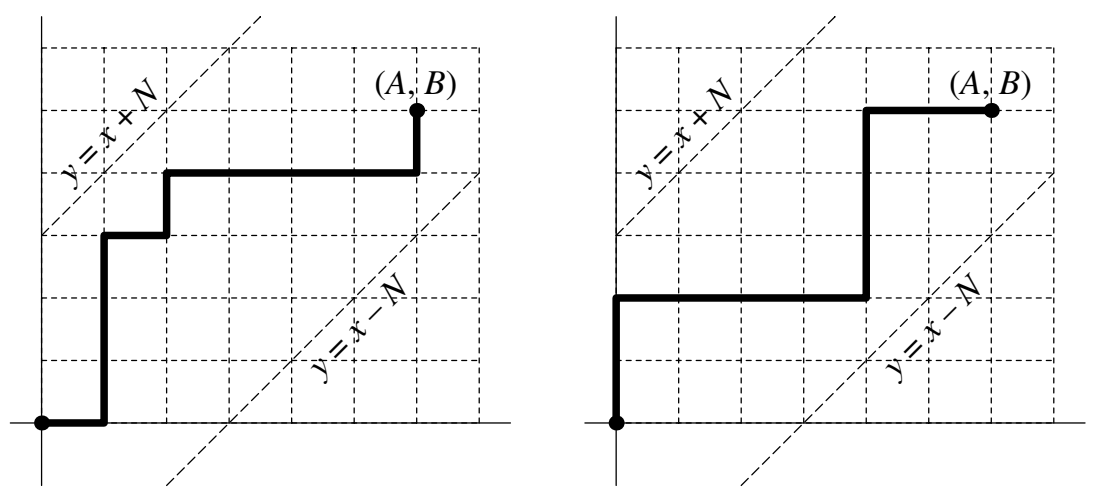

Figure 9. Lattice paths from $(0,0)$ to $(A, B)$ not touching $y=x \pm N$.

The statement of the Lattice Lemma can be found in [10, Proposition 1] and [12, Lemma 4A]. Not mentioned there is a stronger statement, which we need, concerning strict positivity. Namely, we need the following consequence of the Lattice Lemma.

Corollary 1. $\mathcal{L}(A, B)>0$ if and only if $N>1$ and $|A-B|<N$.

That the sum in (7) is zero when $N=1$ is clear because the lines $y=x \pm 1$ are too close together-any lattice path leaving the origin must touch one of the two lines. (Or one could note that the sum is the binomial expansion of $(1-1)^{A+B}$.) In the case that $|A-B|=N$, the point $(A, B)$ is on one of the lines $y=x \pm N$, so there are no admissible paths in that case either. Finally, assuming $N>1$ and $|A-B|<N$, it is easy to construct at least one path from $(0,0)$ to $(A, B)$ that misses the lines $y=x \pm N$, showing that $\mathcal{L}(A, B)$, and therefore the sum in (7), is positive.

To complete the proof of the Cheese Pizza Theorem, we apply the Lattice Lemma and its corollary to the sums in (6).

Beginning with the sum

$$
\sum_{u \in \mathbb{Z}}(-1)^{u}\left(\begin{array}{c}
k \\
\frac{k-q+N}{2}-u N
\end{array}\right)
$$


in (6), let $A=\frac{k-q+N}{2}$ and $B=k-A$. Since $0<N-q<N$, we see that $A>0$ and $|A-B|=|N-q|<N$. If we also have $q>N-k$, then $B>0$ and the conditions of the Lattice Lemma and its corollary are met and (8) is positive. So now assume $q \leq N-k$. First we note that $\left(\frac{k-q+N}{2}-u N\right)>0$ if and only if $0 \leq \frac{k-q+N}{2}-u N \leq k$. Rearranging and applying our assumption that $q \leq N-k$ leads to

$$
0 \leq \frac{-k-q+N}{2} \leq u N \leq \frac{k-q+N}{2} \leq N-q<N
$$

But here $0 \leq u N<N$ leaves $u=0$ as the only possibility, which in turn implies that $q=N-k$. The sum (8) then reduces to a single, positive term if $q=N-k$; otherwise, if $q<N-k$, the sum has no nonzero terms. So (8) is positive if and only if $q \geq N-k$.

Now consider the sum

$$
\sum_{v \in \mathbb{Z}}(-1)^{v}\left(\begin{array}{c}
n-k \\
\frac{n-k-q}{2}-v N
\end{array}\right)
$$

in (6). Proceeding exactly as for (8), one shows that (9) is positive if and only if $q \leq$ $n-k$. (This actually follows by applying a pretty symmetry between (9) and (8), seen by letting $k=n-k^{*}, q=N-q^{*}$, and $u=-u^{*}$ in (8).)

Combining our conclusions about (8) and (9), we have that (8) and (9) are simultaneously positive if and only if $N-k \leq q \leq n-k$. This will be true for (at least) the particular case of $q=N-k$ if $n \geq N$. In that event, for $q=N-k$, we have $q \not \equiv k$ $(\bmod 2)$ and $0<N-k<N$, so this value of $q$ is represented in the sum in (6). Therefore, $\operatorname{since} \sin (q \pi / N)>0$ for each $q \in(0, N)$, there will be at least one positive term in the sum (6) when $n \geq N$, so (6) will be positive if $n \geq N$.

We have shown that for odd $n$, odd $N \geq 3$, and $0<k<n, W=W(N, n, k)$ is strictly positive when $n \geq N$ and is zero when $n<N$. This completes the proof of the Cheese Pizza Theorem.

4. THE DINNER MENU. Pizza has made for a very filling lunch, but we need to consider our next meal(s). What if the dough is allowed to rise on the unit pan that contains our pizza? What if we fill the inside? It turns out that some easy extensions of what we have proven thus far make these possibilities easy to prepare for our party of two. A tray of samples will be presented momentarily.

First, the Opposing Slices Theorem can be generalized in the following way.

Theorem 4 (General Opposing Slices Theorem). For a region $\mathcal{R}$ mentioned in Theorem 2 , be it a strip, slice, or sector, its area is $\iint_{\mathcal{R}} f d A$, where $f \equiv 1$ on the unit disk. But if $f(r, \theta)=f(r)$ is any fixed, integrable, real-valued function defined on the unit disk whose values depend only on the polar coordinate $r$, then Theorem 2 remains true with the area of each region $\mathcal{R}$ replaced by $\iint_{\mathcal{R}} f d A$.

The proof is in the pictures, as before, with no changes. This lets us contemplate volumes.

To complement this generalization, the fully engorged reader will agree that the following stronger version of the Pizza Sign Theorem has already been proven.

Theorem 5 (General Pizza Sign Theorem). Suppose that $s(r)$ is a function represented by a power series convergent on $[-1,1]$. Assume also that the coefficients of 
the series are eventually of like sign. More precisely, suppose that for some odd $N \geq 1$,

$$
s(r)=\sum\left\{b_{n} r^{n}: 1 \leq n<N, n \text { odd }\right\}+\sum\left\{c_{n} r^{n}: n \geq N, n \text { odd }\right\},
$$

where the b's and c's are constants such that one of the following holds: (i) all the c's are 0; (ii) all the c's are $\geq 0$ and at least one is $>0$; (iii) all the c's are $\leq 0$ and at least one is $<0$. Then the sum

$$
(-1)^{\frac{N-1}{2}} \sum_{j=1}^{N}(-1)^{j-1} s\left(R \sin \left(\frac{j \pi}{N}-\alpha\right)\right)
$$

is respectively zero, positive, or negative if (i), (ii), or (iii) holds.

Thus if $s(r)$ is an odd polynomial of degree $M$, then the sum (11) is zero whenever $N>M$. If $s(r)$ has an infinite, odd power series that is not a polynomial, but whose terms $c_{n} r^{n}$ are of like sign (or zero) for $n \geq M$, then if those signs are positive (respectively, negative), the sum (11) will be positive (respectively, negative) for $N \geq M$. We note that the first sum (of the $b$ 's) in (10) is empty (zero) if $N=1$.

Calzones. To us, a calzone is a three dimensional pizza stuffed with cheese. We have adopted the term "calzone" from [15] (which in turn cites [2]), where a nice theorem about slicing spheres in a slightly different way is mentioned. Our calzones are formed by stuffing the space above the unit pan and below a surface given by a nonnegative, radially symmetric function $z=f(r)$. We also assume that $f$ is essentially nontrivial, for example, by requiring that $\int_{0}^{1} f(r) d r>0$. Given a point $P$ in the unit pan, we now cut our dishes using $N$ cutting planes that are perpendicular to the pan and that pass through the original cutting lines containing $P$ (still making $2 N$ equal angles at $P)$. We again alternately color adjacent "slices" gray and white so that the center of the pan lies under a gray slice. By the General Opposing Slices Theorem, we need only concern ourselves with volumes above the central strips, these volumes taking the form of the more general strip function

$$
s(r)=\iint_{s t r(L)} f d A,
$$

where $\operatorname{str}(L)$ is our original central strip of width $r$.

Entrée 1 (Stuffed Paraboloid Caps). Let $f(r)=H\left(1-r^{2}\right)$, for $H>0$ (the height of the cap). It is not difficult to verify that the corresponding strip function is

$$
s(r)=\iint_{s t r(L)} f d A=\frac{4 H}{3}\left(r-\frac{r^{3}}{3}+\sum\left\{c_{n} r^{n}: n \geq 5, n \text { odd }\right\}\right),
$$

where all the c's are positive. This implies that for the paraboloidal calzone, if $N=7,11, \ldots$, then the gray slices have lesser combined volume, while if $N=$ $5,9,13, \ldots$, the gray slices have greater volume.

The stuffed paraboloid is essentially "anti-pizza" for odd $N \geq 5$ in terms of who gets the most to eat. 

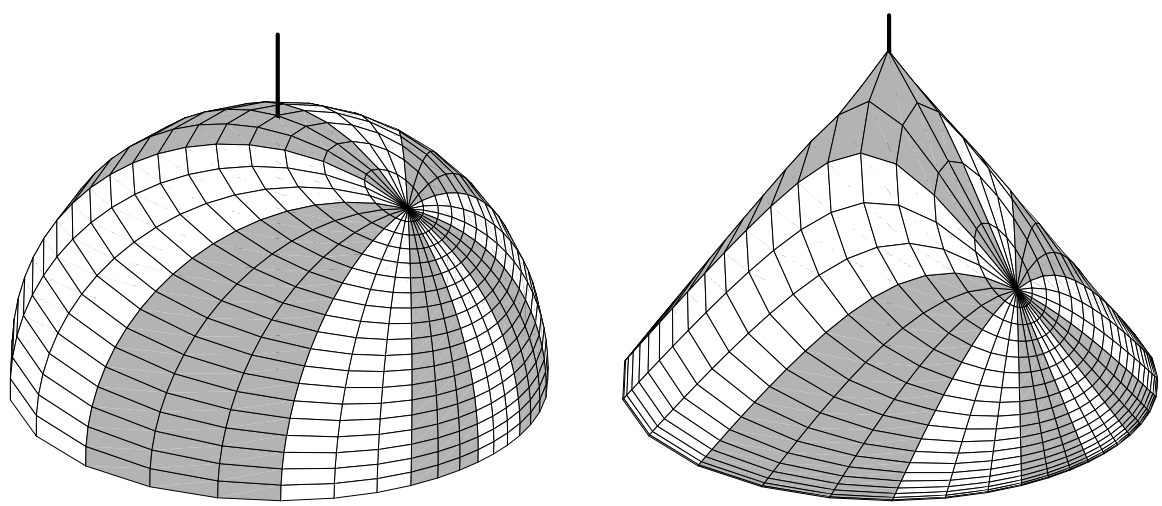

Figure 10. Hemisphere and cone calzones with $N=5$. The $z$-axis is shown poking through, to indicate the slice containing the center.

Entrée 2 (Stuffed Semi-Ellipsoid). Let $f(r)=H \sqrt{1-r^{2}}$, for $H>0$ (the height of the semi-ellipsoid). Then

$$
s(r)=\frac{H \pi}{2}\left(r-\frac{r^{3}}{3}\right) .
$$

Therefore, for the semi-ellipsoidal calzone, the gray slices have greater volume if $N=$ 3 , while for odd $N \geq 5$, the gray volume equals the white!

Crusts and Skins. Some people like to munch on pizza crusts, potato skins, and other fine items. Consider the "thin skin" of a calzone, sliced according to our usual rules. This time we want to calculate the difference in the surface areas of alternate slices of our surface $z=f(r)$. Analogously to our formula for sliced volumes, for these delicacies we want $s(r)$ expressing the polar form of surface area, namely,

$$
s(r)=\iint_{\operatorname{str}(L)} \sqrt{1+\left(f^{\prime}\right)^{2}} d A .
$$

Confection 1 (Thin Skin of Cone). Let $f(r)=H(1-r)$, where $H>0$. Then

$$
s(r)=\sqrt{H^{2}+1}\left(r \sqrt{1-r^{2}}+\sin ^{-1}(r)\right) .
$$

This is equal to $\sqrt{H^{2}+1} s_{c}(r)$, where $s_{c}(r)$ is the strip function given by (3) for the original cheese pizza. So sliced thin crusts of cones are "pizza-like"-the same as the cheese pizza-in terms of who gets the most.

The next is a house specialty. For such a simple creation, it is truly surprising how satisfying it is.

Confection 2 (Thin Crust of Hemisphere). Let $f(r)=\sqrt{1-r^{2}}$. Then the surface area over the strip is

$$
s(r)=\pi r .
$$

For odd $N \geq 3$, the thin crust of a hemisphere gives equal surface areas for both gray and white! 
We mustn’t forget plain old pizza crusts.

Confection 3 (Thin Crust of Pizza). Let $N \geq 2$ and slice the pizza exactly as in the Cheese Pizza Theorem, with the center $O$ not on a cut. Rather than summing areas (cheese) of alternating slices, sum the corresponding arc lengths (thin crust) of those same slices. Then (a) for even $N$, gray and white get the same amount of thin crust, (b) gray gets the most thin crust if $N=5,9,13, \ldots$, and (c) for $N=3,7,11, \ldots$, gray gets the least. (Thus for odd $N \geq 3$, the set of slices having more cheese has less thin crust.)

Proof. When $N$ is even, the proof becomes clear from the illustrations in Figures 5 and 6. With even $N$ we are adding the crusts (arc lengths) of the opposing slices. In Figure 5 for example, since $\left|\widehat{A A^{\prime}}\right|=\left|\overparen{B B^{\prime}}\right|,\left|\widehat{C C^{\prime}}\right|=\left|\overparen{D D^{\prime}}\right|$, and $\left|\widehat{A^{\prime} C^{\prime}}\right|=\left|\widehat{B^{\prime} D^{\prime}}\right|$, we have

$$
|\overparen{A C}|+|\overparen{B D}|=\left|\widehat{A^{\prime} C^{\prime}}\right|+\left|\widehat{B^{\prime} D^{\prime}}\right|=2\left|\widehat{B^{\prime} D^{\prime}}\right|=2 \theta
$$

where $\theta=\angle B^{\prime} O D^{\prime}$. Here $\theta=\pi / N$, so summing over all $N / 2$ pairs of opposing crusts associated with either of the colored sets shows that each person gets the same amount $(\pi)$ of thin crust. (The result for Thin Crust of Pizza in the cases of even $N \geq 4$ is mentioned without proof in [9] and [15].)

For odd $N$, the Opposing Slices Theorem works with arc lengths in place of areas corresponding to the same strips, sectors, and slices, as the reader can easily check. We may then apply Theorem 5 to the function $s(r)$ that gives the arc length of the boundary of a strip on the unit circle, namely,

$$
s(r)=2 \sin ^{-1}(r),
$$

whose power series consists of only odd powers with positive coefficients. This means that $s(r)$ satisfies case (ii) in Theorem 5 for every odd $N \geq 3$. The opposite sign of the coefficients in $s$ compared with that for the cheese pizza function $s_{c}$, which has negative coefficients for odd $N \geq 3$, is the reason that the set of slices having the most crust contains the least amount of cheese for odd $N \geq 3$.

Leftovers. Of course, real whirled pizzas do not have (one-dimensional) thin crust as in the previous confection. More applicable to real pizza crusts is the following.

Leftovers 1 (Thick Crust of Pizza). Let a unit pizza have an inner disk (of radius $c<1$ ) of uniformly distributed cheese with an outer ring (of thickness $1-c$ ) of uniformly distributed crust, each centered at the origin. Let $N \geq 3$ (odd or even) and slice the pizza exactly as in the Cheese Pizza Theorem, but with $P$ located in the cheesy part. Divvy up alternating slices as usual. For $N$ odd, the set of slices giving the most cheese has the least crust. For $N$ even, the amounts of crust are equal.

Proof. We're really just removing one pizza from another and looking at what's left over. The result for even $N>2$ is immediate from Theorem 1, since it implies that the original sliced disk and the inner sliced disk have equal gray and white areas. (See Figure 11 with $N=6$.) For odd $N$, invoke the General Opposing Slices Theorem and Theorem 5 with the function

$$
s(r)=s_{c}(r)-c^{2} s_{c}(r / c),
$$




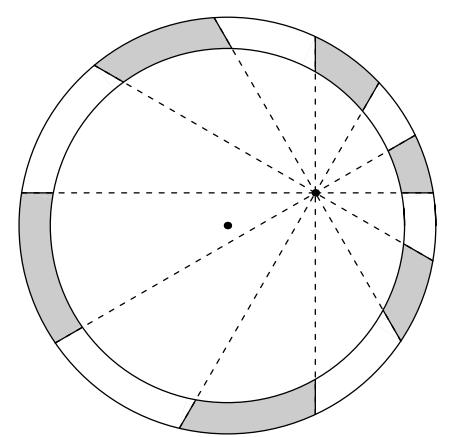

Figure 11. Thick crust with $N=6$ and $c \approx 6 / 7$.

which again satisfies case (ii), having only positive coefficients in its power series expansion.

We left out the cases $N=1,2$ in the Thick Crust of Pizza for seemingly obvious reasons, as these are exceptions in the Cheese Pizza Theorem. The case for $N=1$ is again obvious (gray wins). But it is not hard to show gray equals white when $N=2$ by considering Figure 2 and noting that the rectangle lies in the cheesy part.

We want to include just one more dish (take it home for later), a 3-D version of the thick crust pizza.

Leftovers 2 (Thick Crust Semi-Ellipsoid). Take the semi-ellipsoid in Entrée 2 and make a thick calzone crust between two similar semi-ellipsoids. More precisely, for a fixed $c \in(0,1)$ and $H>0$, this is the region of space between the ellipsoids $z=$ $H \sqrt{1-r^{2}}$ and $z=H \sqrt{c^{2}-r^{2}}$ and for which $z \geq 0$. The gooey part of the calzone is the rest of the semi-ellipsoid, $0 \leq z<H \sqrt{c^{2}-r^{2}}$. We place the cutting point $P$ on the pan $(z=0)$ in this region and slice as usual. Then the gray and white volumes of crust are equal for odd $N \geq 3$.

Proof. If $s_{e}(r)$ denotes the strip function of Entrée 2, then the strip function for this thick-crusted semi-ellipsoid is

$$
s(r)=s_{e}(r)-c^{3} s_{e}(r / c)=\frac{H \pi}{2}\left(1-c^{2}\right) r .
$$

5. CONCLUSION (FOOD FOR THOUGHT). We make a few scattered remarks before going home to sleep off our feast.

First, our calzones are still only half-baked here, as we have not dealt with the cases with even $N$. Many special cases can be easily handled, but for some of the others, the technical difficulty is that we lack a result as simple as the Opposing Slices Theorem when $N$ is even.

There are many other shapes to consider for odd $N \geq 3$. Some have solutions that are simply too long to print in this space (e.g., thin skins of ellipsoids with $H<1$ ). But others do not seem to fall into a category solvable directly by our techniques here. The cheese-filled cone, for instance, has a strip function whose expansion contains an $r^{3} \log r$ term, so we need a modification of our tricks or an ad hoc method to deal with that.

We haven't said much about the case $N=1$, but although it is easy to see that gray always wins in that case, it doesn't necessarily follow as a result of the Pizza Sign Theorem, since the linear term in the power series for $s(r)$ often has a sign opposite those 
in the tail. Careful reading shows that we used the condition $N>1$ early in the proof of the Cheese Pizza Theorem, but nonetheless, the General Pizza Sign Theorem remains true for $N=1$. Our examples with thin and thick crusts illustrate this behavior, as their strip functions have no negative terms.

We left the case $N=3$ unresolved in Entrée 1, but it turns out that when $N=3$ we can say something fairly general. Let $F$ denote the integrand of the strip function in (12) or (13), that is, $F=f$ for volume, $F=\sqrt{1+\left(f^{\prime}\right)^{2}}$ for surface area. The result is that if $F$ is a nonincreasing, nonnegative function on $[0,1]$, then gray $\geq$ white when $N=3$, the inequality being strict so long as $F$ is essentially nontrivial. The proof we like best is a rather obvious adaptation of the visual proof given for $N=3$ in [5]. We leave the details to the truly ravenous reader.

The authors have enjoyed sharing the foodstuffs here (since 1994!), but what happens if others show up for the next meal? Results are known for multi-sharing of cheese pizzas with equiangular slices when each of $M$ people get four slices (see, e.g., [9]), while Rabinowitz proves a version in [15] in which each person gets an even number, four or greater, of such slices.

On that note, we wish to give special thanks and acknowledgement to Stanley Rabinowitz for sharing copies of his manuscript [15], as it contains a veritable smorgasbord of results and problems related to slicing and sharing (in some exotic ways) of pizzas and polygons, among other things. Our new techniques and minor variations of them seem to apply to some of the problems in [15], many of which are as yet solved only for even $N$, so our little diner may be in business for a good while yet. Bon appétit!

\section{APPENDIX.}

Proof of Lemma 1. We provide only a brief outline of the proof (else this appendix would burst).

First we use elementary (albeit laborious) calculations to take a sum of products of powers of sines of different angles and render it, well, powerless. We liberally use symmetry of the binomial coefficients in what follows.

Using $\sin (\theta)=\left(e^{i \theta}-e^{-i \theta}\right) /(2 i)$ along with the binomial theorem, then reversing the order of summations and summing the finite geometric sum (with ratio $-e^{-i(n-2(m+l)) \pi / N}$ ) that appears, we transform (5) into

$$
W=\frac{(-1)^{\frac{N+3}{2}}}{(2 i)^{n}} N e^{i \frac{(n-k) \pi}{N}} \sum_{Q \in \mathbb{Z}} \sum_{(m, l) \in A(Q)}\left(\begin{array}{c}
k \\
m
\end{array}\right)\left(\begin{array}{c}
n-k \\
l
\end{array}\right)(-1)^{m+l} e^{-i 2 l \pi / N},
$$

where $A(Q)=\{(m, l) \mid 0 \leq m+l \leq n$ and $n-2(m+l)=(2 Q+1) N\}$.

Clearly if $n<N$, then $\bar{A}(Q)$ is empty, so the quantity in (14) is zero when $n<$ $N$. This proves the equality case in the statement of Theorem 3. We assume for the remainder of the proof that $n \geq N$.

For $n \geq N$, let $p_{\max } \geq 0$ be the unique integer such that

$$
\left(2 p_{\max }+1\right) N \leq n<\left(2 p_{\max }+3\right) N .
$$

Decompose the sum over $Q$ in (14) by adding pairs of terms corresponding to $Q=$ $p,-(p+1)$, for each $p \in\left\{0,1, \ldots, p_{\max }\right\}$, to produce a difference of complex conjugate terms. Summing over all these pairs of $Q$ 's, using the oddness of the sine, and reversing the order of summations yields

$$
W=\frac{N}{2^{n-1}} \sum_{m=0}^{k}\left(\begin{array}{l}
k \\
m
\end{array}\right) \sin \left(\frac{\pi(k-2 m)}{N}\right) \sum_{p=0}^{p_{\max }}(-1)^{p}\left(\begin{array}{c}
n-k \\
\frac{n-N}{2}-m-p N
\end{array}\right) .
$$


Next some bite-size chunks of our new sum (15) are gathered and simplified, namely those terms having sines whose arguments correspond to coterminal angles between 0 and $\pi$.

Consider $m$ such that $0 \leq m \leq k$ and $\sin \left(\frac{\pi(k-2 m)}{N}\right) \neq 0$ in (15). (The remaining $m$ will be ignored, as they do not contribute to the value of $W$.) Identifying each angle $\frac{\pi(N-(k-2 m))}{N}$ with a coterminal angle of the form $\pm q \pi / N$, where $q \in(0, N)$, we have

$$
\sin \left(\frac{\pi(N-(k-2 m))}{N}\right)=\sin \left( \pm \frac{q \pi}{N}\right) .
$$

We note that $q$ and $k$ have opposite parity.

For each integer $q \in(0, N)$ we gather those $m$ for which (16) holds, as well as marking the signs of the sines, by letting $m_{q}^{ \pm}=\frac{k \pm q-N}{2}$. Then (16) holds if $m \equiv m_{q}^{ \pm}$ $(\bmod N)$. Applying this to (15), we now have

$$
W=\frac{N}{2^{n-1}} \sum_{\substack{q \in(0, N) \\ q \neq k(\bmod 2)}} \sin \left(\frac{\pi q}{N}\right)\left(T_{q}^{+}-T_{q}^{-}\right)
$$

where

$$
T_{q}^{ \pm}=\sum_{\substack{m \equiv m_{q}^{ \pm} \\
(\bmod N)}}\left(\begin{array}{l}
k \\
m
\end{array}\right) \sum_{p=0}^{p_{\max }}(-1)^{p}\left(\begin{array}{c}
n-k \\
\frac{n-N}{2}-m-p N
\end{array}\right)
$$

Since $m_{q}^{-} \equiv k-m_{q}^{+}$, by re-indexing each summation, we see that

$$
T_{q}^{-}=\sum_{j \equiv m_{q}^{+}}\left(\begin{array}{l}
k \\
j
\end{array}\right) \sum_{p=-p_{\max }}^{0}(-1)^{p}\left(\begin{array}{c}
n-k \\
\frac{n+N}{2}-j-p N
\end{array}\right),
$$

where equivalence $(\equiv)$ from here on means equivalence modulo $N$.

By observing that for each $m \in[0, k]$, the numbers $\frac{n+N}{2}-m-p N$ run the full gamut of possible values over $p$ for which nonzero values of $\left(\begin{array}{c}n-k \\ \frac{n+N}{2}-m-p N\end{array}\right)$ are obtained, we see that

$$
\begin{aligned}
T_{q}^{+}-T_{q}^{-} & =\sum_{m \equiv m_{q}^{+}}\left(\begin{array}{c}
k \\
m
\end{array}\right) \sum_{p=-p_{\max }}^{p_{\max }+1}(-1)^{p-1}\left(\begin{array}{c}
n-k \\
\frac{n+N}{2}-m-p N
\end{array}\right) \\
& =\sum_{m \equiv m_{q}^{+}}\left(\begin{array}{l}
k \\
m
\end{array}\right) \sum_{p \in \mathbb{Z}}(-1)^{p-1}\left(\begin{array}{c}
n-k \\
\frac{n+N}{2}-m-p N
\end{array}\right) .
\end{aligned}
$$

Now we let $m=u N+m_{q}^{+}, v=p-1+u$, and take care with signs to transform (19) into

$$
T_{q}^{+}-T_{q}^{-}=\sum_{u \in \mathbb{Z}}(-1)^{u}\left(\begin{array}{c}
k \\
k-u N-m_{q}^{+}
\end{array}\right) \sum_{v \in \mathbb{Z}}(-1)^{v}\left(\begin{array}{c}
n-k \\
\frac{n-N}{2}-v N-m_{q}^{+}
\end{array}\right) .
$$

Inserting $m_{q}^{+}$and combining equations (17) and (20) completes the proof of Lemma 1. 
1. G. Berzsenyi, The pizza theorem—part I, Quantum 4 (January/February, 1994) 29.

2. _ - The pizza theorem—part II, Quantum 4 (March/April, 1994) 29.

3. L. Carter, J. Duncan, and S. Wagon, Problem 1457, Math. Mag. 67 (1994) 304.

4. L. Carter and S. Wagon, Proof without Words: Fair allocation of a pizza, Math. Mag. 67 (1994) 267.

5. P. Deiermann and R. Mabry, The center of a sliced pizza (solution to problem 1457), Math. Mag. 68 (1995) 312-315.

6. M. Goldberg, Divisors of a circle (solution to problem 660), Math. Mag. 41 (1968) 46.

7. J. Härterich and S. Iwata, Solutions to problem 1325, Crux Math. 15 (1989) 120-122.

8. R. I. Hess, Solution to problem 1535, Crux Math. 17 (1991) 179-181.

9. J. Hirschhorn, M. Hirschhorn, J. K. Hirschhorn, A. Hirschhorn, and P. Hirschhorn, The pizza theorem, Austral. Math. Soc. Gaz. 26 (1999) 120-121.

10. M. Ismail, D. Kim, and D. Stanton, Lattice paths and positive trigonometric sums, Constr. Approx. 15 (1999) 69-81.

11. J. D. E. Konhauser, D. Velleman, and S. Wagon, Which Way Did the Bicycle Go? Dolciani Mathematical Expositions, no. 18, Mathematical Association of America, Washington, DC, 1996.

12. T. Narayana, Lattice Path Combinatorics with Statistical Applications, Mathematical Expositions, no. 23, University of Toronto Press, Toronto, 1979.

13. R. B. Nelsen, Proof without Words: Four squares with constant area, Math. Mag. 77 (2004) 135.

14. C. E. M. Pearce, More on the pizza theorem, Austral. Math. Soc. Gaz. 27 (2000) 4-5.

15. S. Rabinowitz, Ptolemy's Legacy, MathPro Press, Westford, MA, (in preparation).

16. L. J. Upton, Problem 660, Math. Mag. 40 (1967) 163.

RICK MABRY received his Ph.D. from the University of South Florida, Tampa, during the previous millenium. His dissertation advisor was Athanassios G. Kartsatos, who bears some inspiration for this paper, thanks to the many trips to CDB's Pizza and Italian Restaurant that he so generously funded. The Cheese Pizza Theorem is but a partial repayment. Since grad school, Rick has spent most of his waking (and dreaming) hours doing and teaching mathematics at LSUS, photographing wildlife on Sand Beach Bayou, creating absurd computer graphics, and playing drums with various local rock, blues, country, Cajun, bluegrass, and (most recently) big band jazz groups.

Louisiana State University in Shreveport, Shreveport, LA 71115

Richard.Mabry@lsus.edu

PAUL DEIERMANN received his B.A. and B.S. degrees from the University of Missouri-St. Louis and his $\mathrm{Ph} . \mathrm{D}$. from Washington University in St. Louis under James A. Jenkins. Besides eating pizza with his lovely Greek wife Eleni and playing with children Maria and Joseph, he likes to play pool, move big rocks, and exercise. He thanks his parents for their lifelong support and encouragement.

Southeast Missouri State University, Cape Girardeau, MO 63701

pdeiermann@semo.edu 